\title{
A Numerical Study on One-Dimensional Reaction-Diffusion Equation and Fisher's Equation
}

\author{
Faria Ahmed Shami ${ }^{1,2, *}$, Laek Sazzad Andallah ${ }^{2}$ \\ ${ }^{1}$ Department of Mathematics, Bangabandhu Sheikh Mujibur Rahman Science and Technology University, Gopalganj, Bangladesh. \\ ${ }^{2}$ Department of Mathematics, Jahangirnagar University, Savar, Dhaka, Bangladesh.
}

How to cite this paper: Faria Ahmed Shami, Laek Sazzad Andallah. (2020) A Numerical Study on One-Dimensional Reaction-Diffusion Equation and Fisher's Equation. Journal of Applied Mathematics and Computation, 4(4), 137-146.

DOI: $10.26855 /$ jamc.2020.12.005

Received: September 29, 2020

Accepted: October 22, 2020

Published: November 4, 2020

*Corresponding author: Faria Ahmed Shami, Department of Mathematics, Bangabandhu Sheikh Mujibur Rahman Science and Technology University, Gopalganj, Bangladesh; Department of Mathematics, Jahangirnagar University, Savar, Dhaka, Bangladesh.

Email:

fariashami@yahoo.com;

fariashami@bsmrstu.edu.bd

\begin{abstract}
The paper concerns with the numerical solution of one dimensional Reaction-Diffusion Equation (RDE). A finite difference scheme is considered for the numerical solution of the RDE. A specific RDE, called the Fisher's equation is studied and the scheme is implemented for the verification of the convergence behavior of the Fisher's equation. An analytical solution is discussed. The error estimation of the scheme is presented to show the rate of convergence graphically. The stability condition of the Fisher's equation is determined by performing numerical experiment.
\end{abstract}

\section{Keywords}

Reaction-Diffusion Equation (RDE), Fisher’s Equation, Finite Difference Scheme, Nonlinear PDE, Numerical solution

\section{Introduction}

The Reaction-Diffusion equation is a partial differential equation arrived in 1937 with the work of R. A. Fisher on the propagation of dominant gene in different environment and circumstances. Fisher formulated a model equation for the spreading of a favorable gene frequency $u$ in a population [1, 2].

This parabolic type PDE is derived on the principle of mass conservation using Fick's law [3].

Reaction-diffusion equations are conventionally used in chemical physics in order to describe concentration and temperature distributions. Today, reaction-diffusion systems have found many applications ranging from chemical and biological phenomena to medicine (physiology, diseases, etc.), genetics, physics, social science, finance, economics, weather prediction, astrophysics, and so on [4, 5].

The Reaction-Diffusion equation (RDE) is a diffusion equation with a reaction term, which may be a function of population density. The equation is

$$
\frac{\partial u}{\partial t}=D \frac{\partial^{2} u}{\partial x^{2}}+R(u)
$$

where $u$ be the population density function, $D$ be the rate of diffusion/diffusion coefficient.

If $R=0$, then equation is simply the diffusion equation or heat equation.

When we use $(u)=r u(1-u)$, it becomes a special RDE named Fisher's equation, which is 


$$
\frac{\partial u}{\partial t}=D \frac{\partial^{2} u}{\partial x^{2}}+r u(1-u)
$$

where $r$ be the reaction coefficient.

Most of the RDEs appear within sets of nonlinear equations or with nontrivial boundary conditions where analytical solutions are either not possible or appears to be sophisticated and it is very difficult to implement real data into the analytical solution of the RDE. Therefore, it is reliable to use the numerical solution methods for the precise solutions of the RDE where the initial and boundary data can be implemented in a much more efficient way. So we study finite difference method for the numerical solution of the RDE [6-11].

The properties of Fisher's equation have been contrived theoretically by many authors. The analysis of travelling wave solution of Fisher's equation has been studied by many computational approaches. Here we investigate an explicit finite difference scheme for the numerical solution of the general RDE and the Fisher's equation and also discuss the condition of stability of the scheme for a given choice of the diffusion coefficient $D$ and the reaction coefficient $r$.

\section{Analytical Solution of General RDE and Fisher's Equation}

\subsection{Analytic Solution of the General Reaction-Diffusion Equation}

It is quite sophisticated to obtain the analytical solution of the RDE. Here we have some exact solution for the general Reaction-Diffusion equation mentioned in [12]. The IBVP is:

$$
\begin{gathered}
x \in[0, L], \quad t \geq 0, \\
u_{t}=D u_{x x}+f(t, x), \\
u(0, x)=u_{0}(x) \\
u_{x}(t, 0)=u_{x}(t, L)=0
\end{gathered}
$$

The solution is given by,

$$
u(t, x)=\int_{-L}^{L} \frac{1}{\sqrt{4 \pi D t}} \sum_{k=-\infty}^{\infty} e^{-\frac{(2 k+x-y)^{2}}{4 D t}} \tilde{u}_{0}(y) d y+\int_{0}^{t} \int_{-L}^{L} \frac{1}{\sqrt{4 \pi D(t-s)}} \sum_{k=-\infty}^{\infty} e^{-\frac{(2 k+x-y)^{2}}{4 D(t-s)}} \tilde{f}(s, y) d y d s,
$$

where $\tilde{u}: \mathbb{R} \rightarrow \mathbb{R}$ denotes for $x<0$ the evenly mirrored $u$ (thus $\tilde{u}_{x}(t, 0)=0$ ).

\subsection{Analytic Solution of the Fisher's Equation}

We have well-established solutions in $[13,14]$ of the Fisher's equation

$$
\frac{\partial u}{\partial t}=D \frac{\partial^{2} u}{\partial x^{2}}+r u(1-u)
$$

where initial condition

$$
u(x, 0)=\left[\frac{1}{2}-\frac{1}{2} \tanh \left(\frac{x}{2 \sqrt{6}}\right)\right]^{2}
$$

and boundary conditions $\lim _{x \rightarrow-\infty} u(x, t)=1$, and $\lim _{x \rightarrow \infty} u(x, t)=0$

The exact solution of this problem is presented in [13] as

$$
u(x, t)=\left[\frac{1}{2}-\frac{1}{2} \tanh \left(\frac{1}{2 \sqrt{6}}\left(x-\frac{5 t}{\sqrt{6}}\right)\right)\right]^{2}
$$

Next we shall use this exact solution to find numerical solution.

\section{Numerical Solution of General RDE and Fisher's Equation}

We construct a general form of IBVP for the one dimensional Reaction-Diffusion equation:

The equation is given by $\frac{\partial u}{\partial t}=D \frac{\partial^{2} u}{\partial x^{2}}+R(u)$

where

with initial condition

$x \in(a, b), t \in(0, T)$

and boundary conditions

$$
u(x, 0)=u_{0}
$$

In order to develop the scheme, we discretize the space and time. We discretize the time derivative $\frac{\partial u}{\partial t}$ and space de- 
rivative $\frac{\partial^{2} u}{\partial x^{2}}$ in the IBVP (5) at any discrete point $(x, t)=\left(x_{j}, t^{n}\right)$ for $j=1,2, \ldots, M$ and $i=0,1,2, \ldots, N-1$.

We discretize the space: $a=x_{-\frac{1}{2}}<x_{\frac{1}{2}}<x_{\frac{3}{2}}<\ldots \ldots \ldots<x_{l-\frac{1}{2}}=b$

We discretize the time: $t=t^{0}<t^{1}<t^{2}<\ldots \ldots \ldots<t^{N-1}=T$

Let the spatial and temporal grid sizes are: $\Delta x=x_{j+\frac{1}{2}}-x_{j-\frac{1}{2}}$ and $\Delta t=t^{n+1}-t^{n}$

Using Taylor series expansion, the discretization of $\frac{\partial u\left(x_{j}^{n}\right)}{\partial t}$ is obtained by first order forward difference in time:

$$
\frac{\partial u\left(x_{j}^{n}\right)}{\partial t} \approx \frac{u_{j}^{n+1}-u_{j}^{n}}{\Delta t}
$$

Using Taylor series expansion, the discretization of $\frac{\partial^{2} u\left(x_{j}^{n}\right)}{\partial x^{2}}$ is obtained by centered difference in space:

$$
\frac{\partial^{2} u\left(x_{j}^{n}\right)}{\partial x^{2}} \approx \frac{u_{j+1}^{n}-2 u_{j}^{n}+u_{j-1}^{n}}{(\Delta x)^{2}}
$$

Now substituting these values in equation (5), we obtain

where $\gamma=\frac{D \Delta t}{(\Delta x)^{2}}$

$$
\begin{aligned}
& \frac{u_{j}^{n+1}-u_{j}^{n}}{\Delta t}=D \frac{u_{j+1}^{n}-2 u_{j}^{n}+u_{j-1}^{n}}{(\Delta x)^{2}}+R\left(u_{j}^{n}\right) \\
& \quad \Rightarrow u_{j}^{n+1}=(1-2 \gamma) u_{j}^{n}+\gamma\left(u_{j+1}^{n}+u_{j-1}^{n}\right)+\Delta t R\left(u_{j}^{n}\right)
\end{aligned}
$$

which is the Explicit Central Difference Scheme (ECDS) and it is also known as FTCS technique.

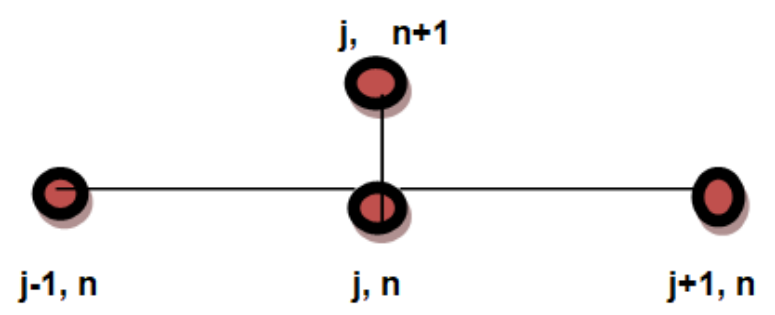

Figure 1. The stencil for the most common explicit method for the general RDE.

\subsection{An Explicit Finite Difference Scheme for Fisher's Equation}

We consider the Fisher's equation as an IBVP

$$
\frac{\partial u}{\partial t}=D \frac{\partial^{2} u}{\partial x^{2}}+r u(1-u), \quad x \in(0,1), t \in(0, T]
$$

with initial condition $u(x, 0)=f(x)$

and boundary conditions $u(0, t)=u(1, t)=0$

Now we apply Explicit Central Difference Scheme on equation (7),

where $\gamma=\frac{D \Delta t}{(\Delta x)^{2}}$.

$$
\begin{aligned}
\frac{u_{j}^{n+1}-u_{j}^{n}}{\Delta t} & =D \frac{u_{j+1}^{n}-2 u_{j}^{n}+u_{j-1}^{n}}{(\Delta x)^{2}}+r u_{j}^{n}\left(1-u_{j}^{n}\right) \\
& \Rightarrow u_{j}^{n+1}=(1-2 \gamma) u_{j}^{n}+\gamma\left(u_{j+1}^{n}+u_{j-1}^{n}\right)+r \Delta t u_{j}^{n}\left(1-u_{j}^{n}\right)
\end{aligned}
$$

Which is an explicit finite difference scheme for Fisher's equation.

\subsection{Stability Condition}

When $r=0$, equation (7) becomes the diffusion equation. Then for this diffusion equation, using (8), the Explicit Central Difference Scheme (ECDS) becomes,

$$
u_{j}^{n+1}=(1-2 \gamma) u_{j}^{n}+\gamma\left(u_{j+1}^{n}+u_{j-1}^{n}\right)
$$


where $\gamma=\frac{D \Delta t}{(\Delta x)^{2}}$.

The ECDS (9) is stable for $0 \leq \gamma \leq \frac{1}{2}$

The equilibrium positions for the logistic equation is well known based on [15, 16]:

The solution $u=\frac{1}{1+A e^{-t}}$ where $A=\frac{1-u_{0}}{u_{0}}$ of the logistic equation

$\frac{d u}{d t}=u(1-u)$ is stable depending on both $u$ and $r$.

- It is equilibrium at $u_{e}=0,1$.

We write, $u_{n+1}=f\left(u_{n}\right)=u_{n}+u_{n}\left(1-u_{n}\right)$

It is stable when $0<f^{\prime}\left(u_{e}\right)<1$

- If $0<r<1$, then the solution of the discrete logistic model monotonically approaches the equilibrium, $u_{e}=1$.

If $1<r<2$, then the solution of the discrete logistic model oscillates about the equilibrium, $u_{e}=1$, but the solution still asymptotically approaches this equilibrium.

If $2<r<3$, then the solution of the discrete logistic model oscillates about the equilibrium, $\boldsymbol{u}_{\boldsymbol{e}}=\mathbf{1}$, but the solution grows away from this equilibrium. ( $r>3$ results in negative solutions).

\section{Error Discussion}

\subsection{A Comparison of Analytical and Numerical Solution}

Here we use the scheme (8) to find the comparison between the analytical and the numerical solution of Fisher's equation. Hence we find how much error is contained in the scheme.

We plot the analytical and numerical solution which we mentioned in equation (4) for different time steps. We combine these two figures to see how the analytical and numerical solutions differ with each other. To mark them separately we use two different colors.

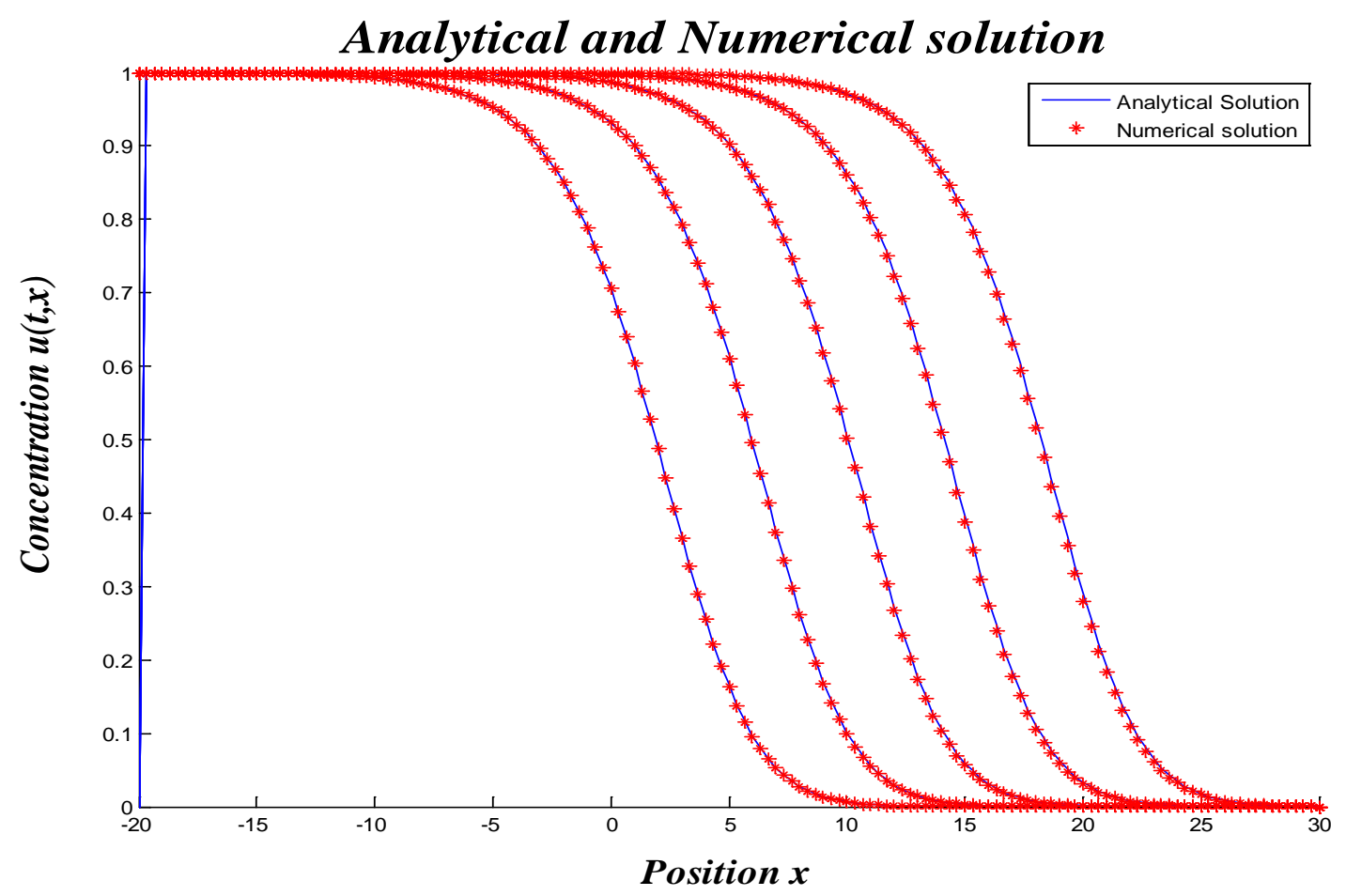

Figure 2. Analytical and Numerical solution of Fisher's equation.

From Figure 2, we observe that the solutions are almost superimposed. Hence we see that the scheme has very less error. Now we shall try to find the absolute error numerically.

\subsection{Error Estimation and Convergence}

We define the following relative error in $\mathrm{L}_{1}$-norm 


$$
\text { err }=\frac{\| \text { Exact solution }- \text { Numerical solution } \|_{1}}{\| \text { Exact solution } \|_{1}}
$$

Then the errors for different temporal and spatial step sizes are computed as demonstrated in the following Figure 3. The figure shows a very good rate of convergence of the explicit finite difference scheme.

As we derive the scheme by Taylor's Series approximation, the scheme is consistent. We also found the scheme is conditionally stable. Therefore, our scheme is conditionally convergent as observe in Figure 3.

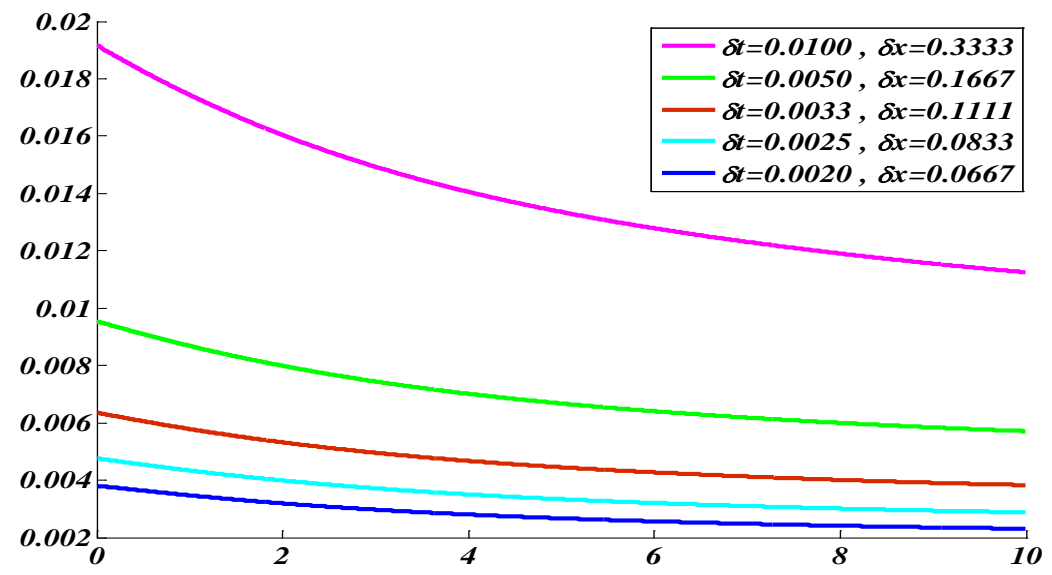

Figure 3. Convergence of the Numerical Scheme.

\subsection{Error Analysis}

From Figure 3, we observe that as time increases, the error of the scheme decreases. We present it briefly in Table 1.

Table 1. Comparison of errors of the scheme at different time steps

\begin{tabular}{|c|c|c|c|c|}
\hline$\Delta \mathbf{t}$ & $\Delta \mathbf{x}$ & Error at starting time, $e r r_{s}$ & Error at ending time, $e r r_{e}$ & Error reduces in the time step, $\left(e r r_{s}-e r r_{e}\right)$ \\
\hline 0.0100 & 0.3333 & 0.01917 & 0.01125 & 0.00792 \\
\hline 0.0050 & 0.1667 & 0.009541 & 0.005713 & 0.003828 \\
\hline 0.0033 & 0.1111 & 0.006351 & 0.00383 & 0.002521 \\
\hline 0.0025 & 0.0833 & 0.004759 & 0.002883 & 0.001876 \\
\hline 0.0020 & 0.0667 & 0.003805 & 0.002312 & 0.001493 \\
\hline
\end{tabular}

So, when we go through Figure 3 and Table 1, we observe that error reduces with the increase of time. After each time step error is reduced than that was at the start of that time step. Thus the scheme becomes more accurate with time.

So we can assume that the explicit central difference scheme will be helpful to find the numerical results almost accurately if we apply it on Fisher's equation. Next we will implement the scheme on the Fisher's equation to find out its numerical behavior.

\section{Numerical Results on Fisher's Equation}

Using scheme (8) for Fisher's equation, we produce numerical results and verify the convergence of Fisher's equation. By performing numerical experiment we report about to choose the pair of parameters diffusion coefficient $D$ and the reaction coefficient $r$ for maintaining stability of the scheme.

\subsection{Convergence of the Solution}

We discuss this convergence based on $[17,18]$. We consider the dimensionless Fisher's equation

$$
\frac{\partial u}{\partial t}=\frac{\partial^{2} u}{\partial x^{2}}+u(1-u)
$$

When we apply the scheme (8), the new scheme for (10) takes the form

$$
u_{j}^{n+1}=(1-2 \gamma) u_{j}^{n}+\gamma\left(u_{j+1}^{n}+u_{j-1}^{n}\right)+\Delta t u_{j}^{n}\left(1-u_{j}^{n}\right), j=1, \ldots, m
$$


where $\gamma=\frac{\Delta t}{(\Delta x)^{2}}$ and $n>0$

with initial condition $u_{j}^{0}=f\left(x_{j}\right), \quad j=0, \ldots \ldots, m+1$ for $n \geq 0$

the boundary conditions $u_{0}^{n+1}=(1-2 \gamma) u_{0}^{n}+2 \gamma u_{1}^{n}+\Delta t u_{0}^{n}\left(1-u_{0}^{n}\right)$

and $u_{m+1}^{n+1}=(1-2 \gamma) u_{m+1}^{n}+2 \gamma u_{m}^{n}+\Delta t u_{m+1}^{n}\left(1-u_{m+1}^{n}\right)$

We compute the numerical solution of the IBVP using the temporal and spatial step size $\Delta t=0.001, \Delta x=0.05$ respectively. The numerical solutions are functions of $x$ for different values of $t$.

We take the initial condition $f(x)=\sin (\pi x)$ when $\Delta t=0.001, \Delta x=0.05$. Then solutions at time $t=0,0.05,0.5,5$ seconds respectively presented as Figure 4.
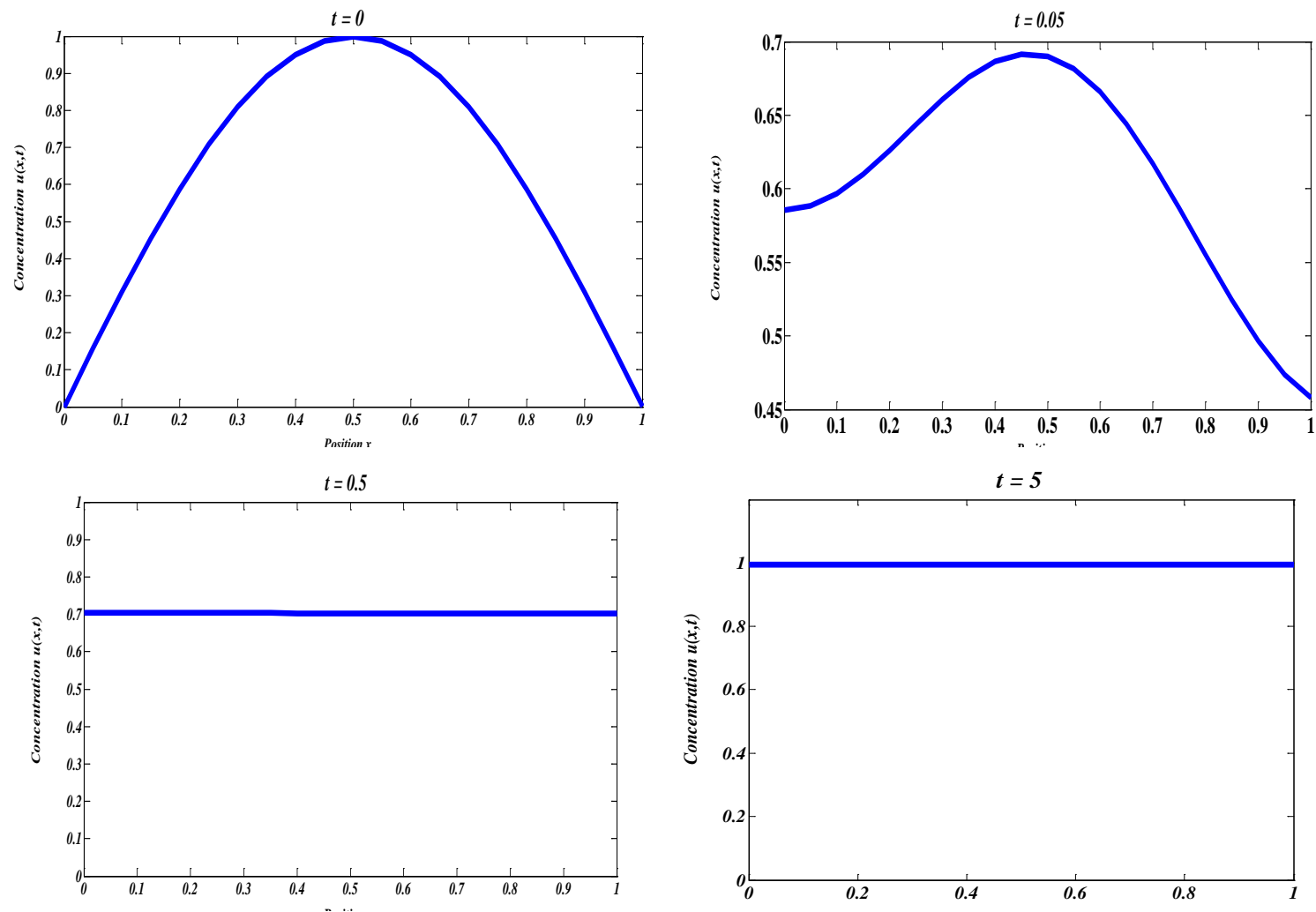

Figure 4. An approximate solution of the model using $f(x)=\sin (\pi x)$ for time $t=0,0.05,0.5,5$ seconds respectively.

We discussed about the equilibrium points of the logistic function. We know that for a large time step the solution oscillates between the equilibrium points and so at the final time, it converges to its equilibrium points.

From figure we observe that, in each case, the approximate solutions seem to remain within the unit interval. Also they seem to converge towards the asymptotic solution $u=1$ for all $x$ as $t$ increases. Thus we can say the unit interval as an invariant region for this model [17].

\subsection{Selecting the Value of Diffusion Coefficient $D$ and the Reaction Coefficient $r$}

Let us construct an IBVP,

where $x \in(0,4), t \in(0,10]$

$$
\frac{\partial u}{\partial t}=D \frac{\partial^{2} u}{\partial x^{2}}+r u(1-u)
$$

with initial condition $u(x, 0)=\sin \left(\frac{\pi x}{2}\right)+1$

and Dirichlet boundary conditions $u(0, t)=u(4, t)=1$

We discuss about the diffusion coefficient $D$ and the reaction coefficient/rate of growth $r$.

According to (8), the discretized scheme for (12) will be, 


$$
u_{j}^{n+1}=(1-2 \gamma) u_{j}^{n}+\gamma\left(u_{j+1}^{n}+u_{j-1}^{n}\right)+r \Delta t u_{j}^{n}\left(1-u_{j}^{n}\right)
$$

where $=\frac{D \Delta t}{(\Delta x)^{2}}$.

From previous discussion, we know for $r=0$, the stability condition is $\gamma \leq \frac{1}{2} \Rightarrow \frac{D \Delta t}{(\Delta x)^{2}} \leq \frac{1}{2}$

Now by numerical experiment we try to understand the effect of the parameter $r$ on the stability condition.

For this we present here graphical representation of different situations of the solution for different values of $D$ and $r$, when $\Delta t=0.01$ and $\Delta x=0.1$.

First we consider the case $D=0.5$ and $r=0$. i.e. the diffusion equation (Figure 5).

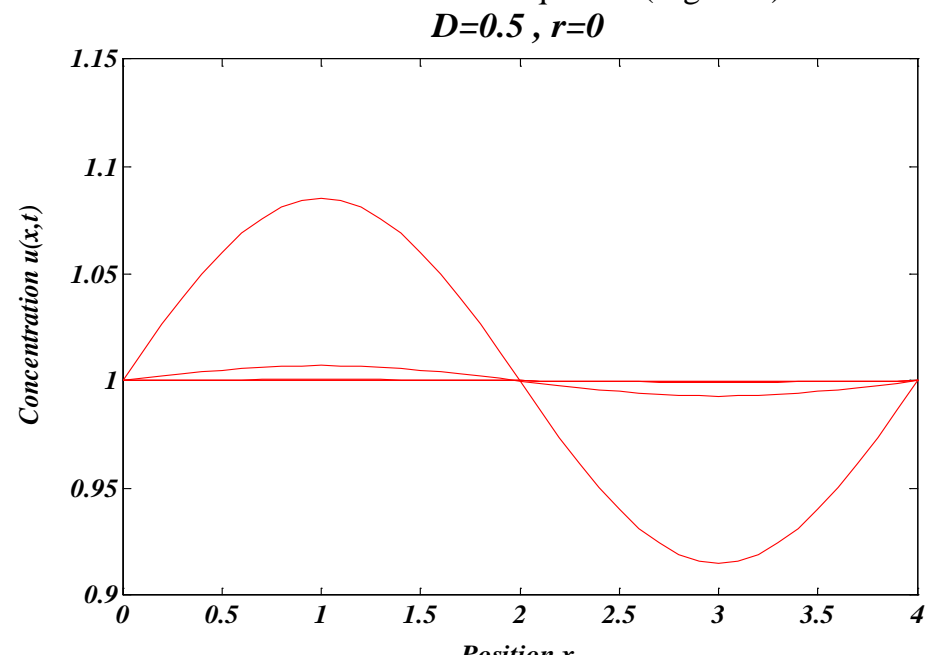

Figure 5. The solution of (12) at different times when $D=0.5, r=0$, at the case $\Delta t=0.01$ and $\Delta x=0.1$.

From figure we see that the diffusion equation is stable at $D=0.5$. i.e. at $\gamma=0.5$

To test if the stability condition is correct we keep the value of $r$ fixed and increase the value of $D$. Here we consider $D=0.51$ and $r=0$ (Figure 6).

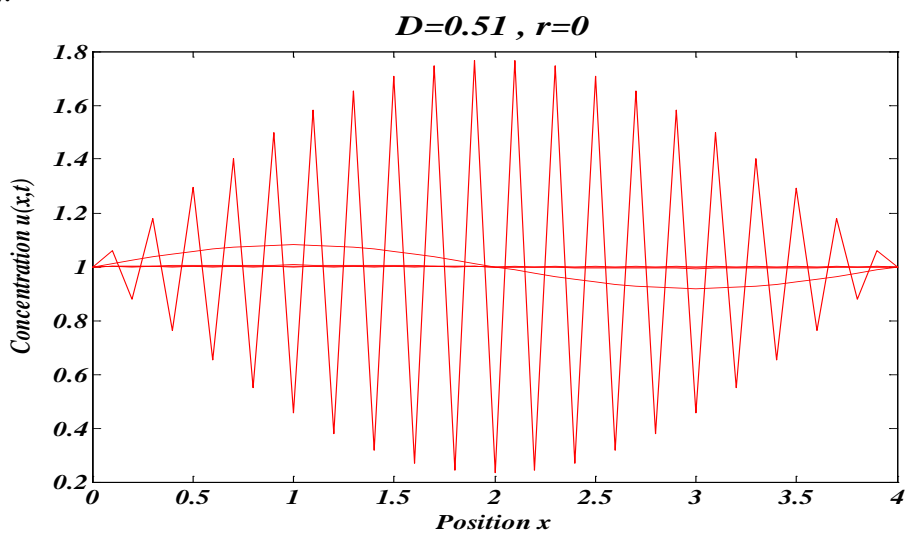

Figure 6. The solution of (12) at different times when $D=0.51, r=0$, at the case $\Delta t=0.01$ and $\Delta x=0.1$.

From figure we see that for $D=0.51$ the solution is unstable. Hence we say that the diffusion equation is stable for $\gamma \leq \frac{1}{2}$.

Now we consider very small case for both $D$ and $r$, which satisfy the condition of stability. Here we consider $D=0.05$ and $r=0.05$ 


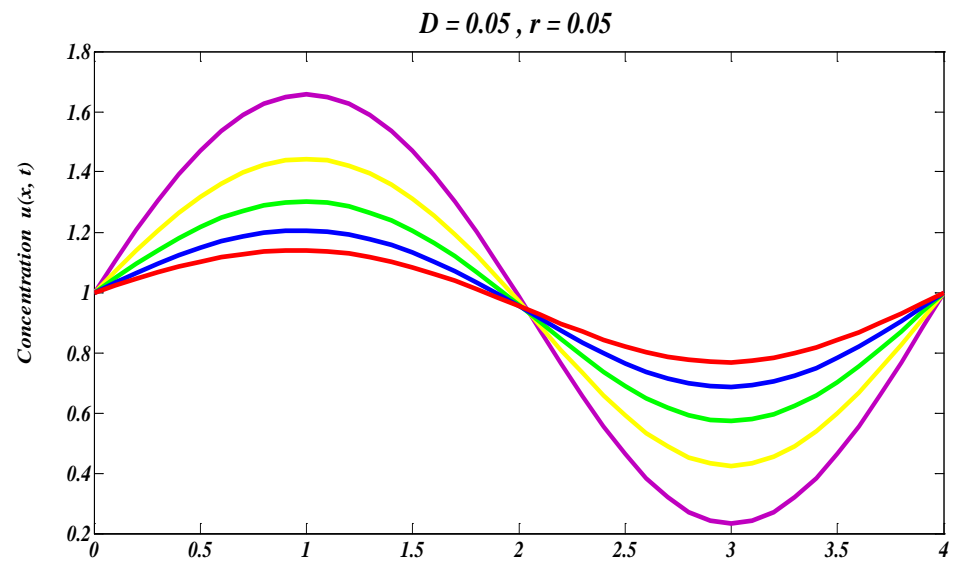

Figure 7. The solution of (12) at different times when $D=0.05, r=0.05$, at the case $\Delta t=0.01$ and $\Delta x=0.1$.

From Figure 7, we see that the solution is stable at $D=0.05$ and $r=0.05$.

Now we consider $D=0.5$ and $r=1$

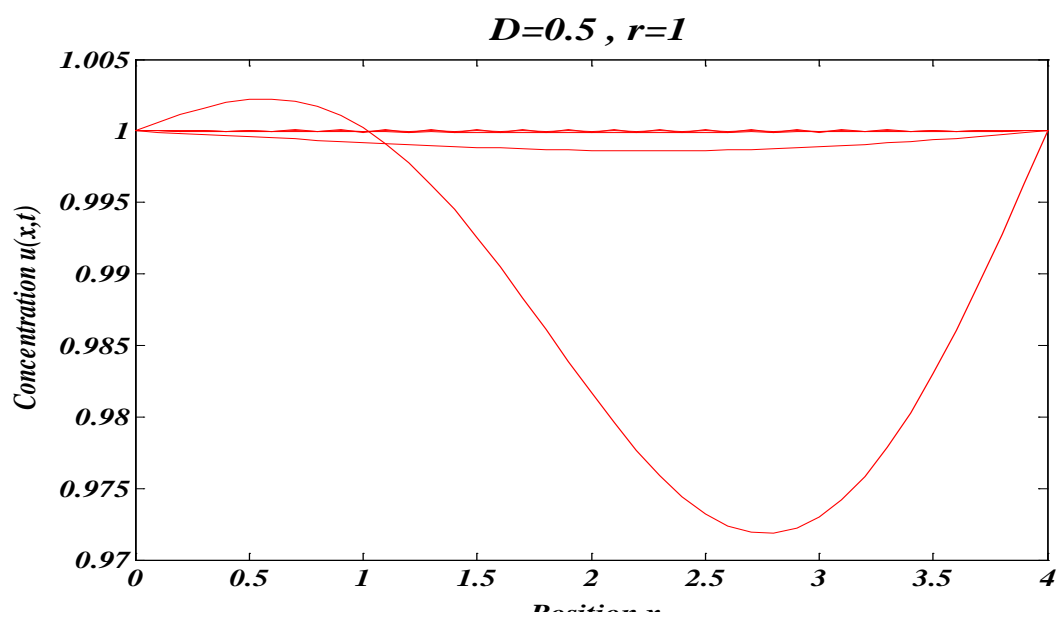

Figure 8. The solution of (12) at different times when $D=0.5, r=1$, at the case $\Delta t=0.01$ and $\Delta x=0.1$.

From Figure 8, we see that the solution starts to be unstable at $D=0.5$ and $r=1$.

Now we consider $D=0.5$ and $r=2$

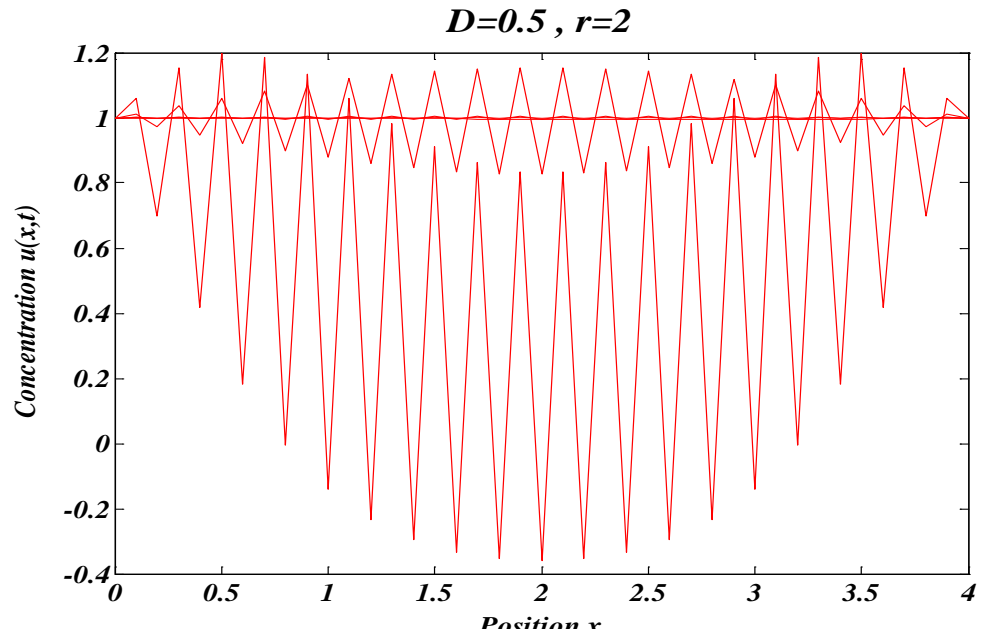

Figure 9. The solution of (12) at different times when $D=0.5, r=2$, at the case $\Delta t=0.01$ and $\Delta x=0.1$. 
From Figure 9, we see that the solution is completely unstable at $D=0.5$ and $r=2$.

From the above discussion, we summarize different stability situations in the following Table 2 when $\Delta t=$ 0.01 and $\Delta x=0.1$,

\section{Remark:}

From Figure 5 to Figure 9 and Table 2, we see that the solution is stable when $\gamma \leq 0.5$. If we increase the value of $\gamma$ in a very small amount, the solution meets the stability for very large values of $r$. Hence we observe that, on stability condition the effect of $r$ is very small.

Therefore, to maintain stability we need to choose $D$ so that $\gamma \leq 0.5$ only and then $r \leq 1$. But in realistic situation, i.e., in logistic population model it is observed that the value arises as $r \cong 0.02$. So this analysis permits us to have a wide range of $r$. Then we will be able to discuss the case when the population growth rate is high and the rate does not affect the stability of population function.

Table 2. Different stability situations for different values of $D$ and $r$

\begin{tabular}{|c|c|c|c|}
\hline $\begin{array}{c}\text { Diffusion Coefficient, } \\
D\end{array}$ & $\begin{array}{c}\gamma=\frac{D \Delta t}{(\Delta x)^{2}} \\
(\gamma=\mathrm{D} \text { in this case })\end{array}$ & Reaction Coefficient, $r$ & Stability \\
\hline 0.52 & 0.52 & $10^{-15}$ & Unstable \\
\hline 0.52 & 0.52 & $10^{-14}$ & Unstable \\
\hline 0.51 & 0.51 & $10^{-4}$ & Unstable \\
\hline 0.51 & 0.51 & $10^{-3}$ & Unstable \\
\hline 0.51 & 0.51 & 0 & Stable \\
\hline 0.5 & 0.5 & 0 & Stable \\
\hline 0.5 & 0.5 & 0.05 & Stable \\
\hline 0.5 & 0.5 & 0.1 & Stable \\
\hline 0.5 & 0.5 & 0.5 & Stable \\
\hline 0.5 & $\underline{0.5}$ & $\underline{1}$ & Starts to be Unstable \\
\hline 0.5 & 0.5 & 2 & Unstable \\
\hline 0.5 & 0.5 & 8 & Unstable \\
\hline 0.5 & 0.5 & 9 & Unstable \\
\hline 0.45 & 0.45 & 9 & Stable \\
\hline 0.45 & 0.45 & 59 & Unstable \\
\hline 0.45 & 0.45 & 69 & Unstable \\
\hline 0.4 & 0.4 & 69 & Stable \\
\hline 0.4 & 0.4 & 90 & Unstable \\
\hline 0.4 & 0.4 & 101 & Unstable \\
\hline
\end{tabular}

\section{Conclusion}

We discussed about the numerical solution using an explicit difference scheme. We estimated the error and the result is accurate up to 4 decimal places and a good rate of convergence is demonstrated graphically.

We verified that the solution of the Fisher's equation converges to its equilibrium solution. We also came to a decision that condition of stability for the explicit central difference scheme for Fisher's equation remains same as that for diffusion equation in case of the realistic choice of the value of the diffusion parameters $D$ and reaction parameter $r$.

In future we want to study on this interesting topic by applying other schemes and want to concentrate on applications like population dynamics or genetics. Using these results we can be able to find the nature and size of population after a certain time with the effects of the natural phenomena and circumstances. 


\section{References}

[1] R. A. Fisher. (1937). “The Wave of Advance of Advantageous Genes”. Annals of Genetics, Vol. 7, No. 4, p. 353.

[2] Logan, J. D. (1994). An Introduction to Nonlinear Partial Differential Equations. Wily-Interscience, New York.

[3] Leveque, R. J. (1992). “Numerical Methods for Conservation Laws”. 2nd Edition, Springer, Berlin.

[4] J. Smoller. (1994). “Shock Waves and Reaction-Diffusion Equations”. 2nd ed, Springer-Verlag.

[5] Canosa, J. (1973). On a nonlinear diffusion equation describing population growth, IBM. J. Res. Dev. 17, $307-313$.

[6] Rohila, R., Mittal, R. C. (2018). Numerical study of reaction diffusion Fisher's equation by fourth order cubic B-spline collocation method. Math Sci, 12, 79-89.

[7] Singh, A., Das, S., Ong, S. H., \& Jafari, H. (2019). "Numerical Solution of Nonlinear Reaction-Advection-Diffusion Equation.” ASME. J. Comput. Nonlinear Dynam. April 2019; 14(4): 041003.

[8] Shahid, N., Ahmed, N., Baleanu, D., Alshomrani, A., Iqbal, M., Rehman, M., Shaikh, T., \& Rafiq, M. (2020). Novel numerical analysis for nonlinear advection-reaction-diffusion systems. Open Physics, 18(1), 112-125.

[9] Gazdag, J., Canosa, J. (1974). Numerical solution of Fisher’s equation. J. Appl. Probab. 11, 445-457.

[10] Parekh, N., Puri, S. (1990). A new numerical scheme for the Fisher's equation. J. Phys. A, 23, L1085-L1091.

[11] Wazwaz, A. M., Gorguis, A. (2004). An analytic study of Fisher's equation by using Adomian decomposition method. Appl. Math. Comput., 154, 609-620.

[12] Dr. Klemens Fellner. (2010). Reactions-Diffusion Equations, University of Cambridge, October 25, 2010.

[13] X. Y. Wang. (1988). “Exact and explicit solitary wave solutions for the generalized Fisher’s equation”. Phys. Lett. A, 131(4-5): 277-279.

[14] Brajesh Kumar Singh and Geeta Arora. (2014). “A numerical scheme to solve Fisher-type reaction-diffusion equations”. MESA Vol. 5, No. 2, pp. 153-164.

[15] Joseph M. Mahaffy. (2009). “Logistic Growth and Nonlinear Dynamical Systems”. San Diego State University, Spring Semester.

[16] Broadbridge, P., Bradshaw-Hajek, B. H. (2016). Exact solutions for logistic reaction-diffusion equations in biology. Z. Angew. Math. Phys. 67, 93.

[17] A. Tveito, R. Winther. (1991). “Introduction to Partial Differential Equations-A Computational Approach”. Springer.

[18] Fellner, K., Tang, B. Q. (2018). Convergence to equilibrium of renormalised solutions to nonlinear chemical reaction-diffusion systems. Z. Angew. Math. Phys., 69, 54(2018). 